\title{
The repairing of full-thickness skin deficiency and its biological mechanism using decellularized human amniotic membrane as the wound dressing
}

\author{
Mengsheng Song ${ }^{1}$, Weiqing Wang ${ }^{1}$, Qihua $\mathrm{Ye}^{1}$, Shizhong $\mathrm{Bu}^{1}$, Zhisen Shen ${ }^{2}$, and Yabin Zhu ${ }^{{ }^{*}}$ \\ ${ }^{1}$ The Medical School, Ningbo University, Ningbo 315211, Zhejiang, China \\ ${ }^{2}$ The Lihuili Hospital Affiliated to the Medical School, Ningbo University, Ningbo 315041, \\ Zhejiang, China
}

\begin{abstract}
Human amniotic membrane (HAM) was a biocompatible scaffold with advantages of anti-inflammatory, low antigen, feasibility, tolerance and low cost. In our previous work, HAM was treated to be decellularized using surfactant, lipase and DNAase methods and the efficacy as an implantable biological mesh was verified after decellularization treatment. In this work, we used the previous protocol to decellularize the fresh HAM, and applied it to repair full-thickness skin defects with Sprague-Dawley rats as the test animals. The wound healing progress was followed in the duration of 8 months, and the biological repairing mechanism was explored. From the wound area alteration, white blood cell (WBC) measurements and H\&E staining, dHAM was detected to promote the wound healing, comparing with the traditional clinic treatment. Immunohistochemical analyses of the bio-factors involved in the wound healing, vascular endothelial growth factor (VEGF), alpha-smooth muscle actin ( $\alpha$-SMA) and transforming growth factor beta-1 (TGF- $\beta 1$ ), exhibited that dHAM enhanced VEGF and $\alpha$-SMA secretion but reduced TGF- $\beta 1$ expression at early stage, which alleviated the wound inflammation, promoted the tissue regeneration and relieved the scar formation.
\end{abstract}


Keywords - Decellularized human amniotic membrane (dHAM); wound healing; mechanism; skin regeneration

\section{INTRODUCTION}

As the largest protective organ, skin plays an important role in defending against harms from external environment [1]. Skin is also the first safeguard of human body, which provides the functions of sensation and water equilibrium [2,3]. Any skin wounds such as burns, chemical damage and tearing apart will cause the body infections, ulcers and ultimately scars after wound repairing [4]. The necessary treatment is to find a wound dressing as early as possible, aiming at healing the defects and restoring the functions of the skin [5]. Currently the traditional methods include autologous skin graft, allogeneic skin transplantation, xenogeneic graft and artificial synthetic skin substitutes [6]. However, due to the donor deficiency, immunological rejection and the possibility of carrying disease, the application of those methods is still limited. Seeking an ideal skin substitute is an urgent task for skin regeneration in clinic [7].

Human amniotic membrane (HAM) is a translucent membrane with the thickness of 0.02-0.5 mm. It consists of three main components, i.e. epithelial layer, basement layer and connective tissue layer [8]. It is increasingly applied to wounds, burns and ophthalmology in recent years due to its advantages of anti-inflammatory, low antigen, feasibility, tolerance and low cost $[9,10]$. In our previous work, HAM has been decellularized using surfactant, lipase and DNAase methods, where surfactant and 
lipase were used to denature cell membrane and DNAase was used to enzymolyze DNA content. The decellularization efficiency and the biocompatibility as an implantable biological mesh after treatment were verified [11]. The decellularized HAM contains only basement layer with the components of collagen types I, III and IV, laminin (LN), fibronectin and a variety of growth factors [12,13]. The LN contains a variety of protein molecules, which composes extracellular matrix (ECM) as non-collagen glycoprotein. It combines with collagen IV to form the ECM skeleton $[14,15]$. Thus, the decellularized human amniotic membrane (dHAM) becomes more biocompatible and is thought to be more suitable as a biological substitute than HAM [11]. On the other hand, the dHAM still contains a variety of growth factors such as epidermal growth factor (EGF), hepatocyte growth factor (HGF), keratinocyte growth factor (KGF), nerve growth factor (NGF), basic fibroblast growth factor (bFGF) and transforming growth factor (TGF) alpha and beta etc. [16,17] All these growth factors have powerful abilities in promoting cell or/and tissue regeneration.

As literatures reported, the progress of wound healing is complex, continuous and dynamic. It usually includes three phases (inflammation, proliferation and remodeling), and involves various cells, cytokines and growth factors which interact with each other to regenerate the whole layers of skin $[18,19]$. Some researches have been conducted with HAM as the wound dressing and the healing effect was evaluated $[20,21]$. In this work, we treated the fresh HAM with our previous protocol to get decellularized HAM. This dHAM was applied to cover the wound as the animal skin was defected. The results displayed that dHAM promoted skin repairing at early 
stage and advanced the skin regeneration in the whole stage. The molecular mechanism was also explored in the animal model, via tracing the wound evolution and detecting the alterations of some growth factors. We thought that the dHAM might influence one or two or all three phases to benefit the healing conditions.

\section{MATERIALS}

Fresh HAM was obtained from Obstetrics Department of Ningbo First Hospital, China. Triton X-100 (Solarbio, China), penicillin and streptomycin (Beyotime Institute of Biotechnology, China), lipase (Solarbio, China) and DNAase (Sinopharm Chemical Reagent Co., China) were utilized in decellularizing process of HAM. Primary antibodies, anti- $\alpha$-SMA (Abcam, China), anti-VEGF (Boster, China) and anti-TGF- $\beta 1$ (Boster, China), goat anti-rabbit IgG (ZSBio PV-6001, China) and 3,3'-diaminobenzidine (DAB, ZSBio, China) were used in immunohistochemical staining.

Sprague-Dawley rats (2 months-old, male) were ordered from Experimental Animal Center of Zhejiang Province and kept in the experimental animal center of Medical School of Ningbo University.

\section{METHODS}

\section{A. Decellularization of HAM}

Decellularization of HAM was performed with our previous protocol [11]. HAM was peeled from placenta by mechanical treatment after blood on the fresh placenta 
was washed away using phosphate buffered saline (PBS). The clean HAM was put into 75\% alcohol/water for $10 \mathrm{~s}$ and rinsed with PBS containing penicillin-streptomycin (200 U/mL) immediately. It was then immersed in PBS (penicillin-streptomycin, $200 \mathrm{U} / \mathrm{mL}$ ) with shaking for $2 \mathrm{~d}$ and changing the solution for each 12 h. Finally, treatments with chemicals in sequence included 1\% Triton X-100 PBS for $4 \mathrm{~h}$, lipase PBS (2000 U/L) for $10 \mathrm{~h}$ and DNAase PBS (2000U/L) for $4 \mathrm{~h}$ at $37^{\circ} \mathrm{C}$.

The whole performance was conducted under the sterile state. The decellularized HAM (dHAM) was cut into pieces around $2.0 \times 2.0 \mathrm{~cm}$ and stored at $4^{\circ} \mathrm{C}$ until the surgery was operated.

\section{B. Full-thickness skin defects on rats'back}

25 rats were collected to be anesthetized with $3 \%$ pentobarbital sodium (intra-peritoneal injection, 30mg/kg). After shaving off the fur and disinfecting the surface, two full-thickness defects at two sides of the rat's back were symmetrically made with the wound size of $1.3 \mathrm{~cm} \times 1.3 \mathrm{~cm}$ (Fig.1a). One piece of $2.0 \mathrm{~cm} \times 2.0 \mathrm{~cm}$ dHAM was used to cover the left wound (Fig.1b, arrow, named as dHAM group) entirely but not the opposite wound which was cleaned by saline (Fig.1b,named as control group). Both wounds were sterilized with iodine, covered with gauze and dressed with medical tapes. All rats were fed normally and kept in cages individually. Each rat was given penicillin (160,000 U/ml) through intra-peritoneal injection once a day at the initial three days after operation. The gauze was removed next day and iodine was applied to disinfect wounds each day within the first week. After some 
days, skins at the wound site were cut off and stored at $-80^{\circ} \mathrm{C}$ for the following tests.

The animals used in this study were treated in accordance with the ethical committee of Ningbo University and NIH’s Principles of Laboratory Animal Care.

\section{Tracing the wound areas}

12 rats were processed as above, aiming to trace the wound area alteration with the time past. The wound areas were recorded at the operation day (1d) and the following each day postoperation. 12 repeats with each group at the each time point were performed and averaged. The healing ratios, wound area at different time/original defect area, were thus calculated. The curves to display the healing tendency were then schematically drawn and the statistical analyses were performed.

\section{Measurements of White blood cells}

Two groups (dHAM group and control group) with 4 rats for each group were designed to detect the white blood cell (WBC) level by hemocyte analyzer (Boehringermannheim BM830, China) after rats were defected. Two wounds with same treatment were made on one rat's back. $0.5 \mathrm{ml}$ blood from caudal veins at $3 \mathrm{~d}, 5$ d, $7 \mathrm{~d}, 9 \mathrm{~d}, 11 \mathrm{~d}$ and $13 \mathrm{~d}$ was collected. The data at the operation day (before the surgery) was recorded as the basic WBC level of the animal $(1 \mathrm{~d})$. The growth rate of WBC was calculated as $\left(\mathrm{N}_{1}-\mathrm{N}_{0}\right) / \mathrm{N}_{0}$, where $\mathrm{N}_{1}$ is the measured WBC value and $\mathrm{N}_{0}$ is the basic level. Four repeats with each group at each time were conducted and averaged. The curves to display the inflammatory tendency were then schematically drawn and statistical analyses were performed.

\section{E. H\&E and immunohistochemical staining}


The regenerating skin tissues around the wounds of rats were obtained separately at $3 \mathrm{~d}, 7 \mathrm{~d}, 14 \mathrm{~d}, 21 \mathrm{~d}, 28 \mathrm{~d}, 42 \mathrm{~d}, 56 \mathrm{~d}$ and 8 mon. After fixed in 4\% paraformaldehyde for $24 \mathrm{~h}$, the samples were sectioned at $4 \mu \mathrm{m}$ on a Microtome (Leica CM1950, Germany). Then, hematoxylin-eosin (H\&E) staining was conducted. The sectioned samples were rinsed with water to remove the opti-mum cutting temperature compound (OCT, Sakura, America) which was used to embed the samples before microtoming. After then, the samples were stained with haematoxylin for $7 \mathrm{~min}$, followed by removal of the excess dye in 1\% hydrochloric acid/alcohol solution for 3 sand immersion in $1 \%$ ammonia solution for $10 \mathrm{~s}$. Subsequently, the samples were rinsed with water and immersed in $0.5 \%$ eosin solution for $10 \mathrm{~s}$ to stain the cytoplasm. After dehydration in ascending series of ethanol for $2 \mathrm{~s}$ each, transparency in dimethylbenzene twice for $3 \mathrm{~s}$ each time, and sealing pieces with neutral gum, light microscopy (Olympus CX40, Japan) was used to capture the H\&E staining images.

For the samples at day 7 and day 14, the immunohistochemical staining was performed separately with the primary antibodies of vascular endothelial growth factor (VEGF) or alpha-smooth muscle actin ( $\alpha$-SMA). Samples at day 3 were detected the expressions of transforming growth factor beta- 1 (TGF- $\beta 1$ ).The standard immunostaining protocol was followed as literature [22] reported. The sectioned samples were rinsed with water, followed by 3\% hydrogen peroxide/deionized water for 5 min and PBS rinsing thrice for 2 min each time. Primary anti- $\alpha$-SMA (dilution 1:250), anti-VEGF (dilution 1:250) and anti-TGF- $\beta 1$ (dilution 1:250) were 
individually incubated overnight at $4^{\circ} \mathrm{C}$. After rinsed with PBS in the same way, the samples were incubated in goat anti-rabbit IgG for $20 \mathrm{~min}$ at $37^{\circ} \mathrm{C}$. And after rinsed with PBS, the samples were stained with DAB (dilution 1:20). The nuclei of the cells in the samples were revealed by stained with hematoxylin for $7 \mathrm{~min}$.

\section{F. Quantitative analyses for VEGF and $\alpha$-SMA staining}

The sectioned samples were stained immunohistochemically as above described. Three pictures (x 200) were selected randomly from its corresponding sample. Using Image-Pro Plus 6.0 software, the integrated option density (IOD) value of each photograph was analyzed. 3 repeats with each group at each time point were performed and averaged. The IOD values of these two groups at each time point were compared and statistically analyzed to discover the expression changes during wound healing.

\section{G. Statistical analysis}

All data were shown as mean \pm standard deviation (SD). Statistical analyses were performed with IBM SPSS Statistics 19. p-Value below 0.05 was considered to be significant.

\section{Results}

\section{A. Healing progress of full-thickness skin wound}

\section{1) Appearance of wound region}

The healing took place after surgery, with secreting blood and tissue fluid at the first several days. Wounds in both groups (control and dHAM) contracted gradually 
towards the wound center. The scar formed at last as shown in Fig.2. Compared with these two groups, wound in dHAM group (Fig.2, arrow) reduced the fluid secretion to form a thinner scab. And, the wound in dHAM group was smaller than that in the control group (Fig.2, no arrow), which was considered to recover faster in dHAM than in control. The both scabs were desquamated at about 17 days. After 21 days post-operation (Fig.2 a3), wound could be still obvious though it was covered by surrounding hair. After cutting off the fur, the scars displayed in both groups as $\mathrm{X}$ shape at day 28, changing from the original square wound shape (Fig.2 a4). No hair grew on the very $\mathrm{X}$-shape scar.

\section{2) The alteration of wound area}

The area of wound was measured and recorded since the operation day (Fig.3a). Generally, areas of both wounds (dHAM and control) reduced rapidly during the first 11 days. After then they changed mildly till to a plateau at day 17. From the tendency curve (Fig.3 b), the wound area of dHAM group was always lower than that of the control group during the first 9 days, implying that rats in dHAM group recovered faster than those in control group. The differences between these two groups at $3 \mathrm{~d}$ $\left({ }^{*} p=0.018\right), 5 \mathrm{~d}\left({ }^{\&} p=0.024\right)$ and $7 \mathrm{~d}\left({ }^{\alpha} p=0.010\right)$ showed the statistical significance.

3) Measurements of WBC level

WBC level is proportional to the wound inflammation in clinic. It was measured for the wounds in both groups. As shown in Fig.4a, WBC values increased after operation, reached the maximum at day 9, and then descended to a stable value. Comparing between these two curves, the value in control group was always higher 
than that in dHAM group. The differences of WBC values between these two groups were significant at $3 \mathrm{~d}\left({ }^{*} p=0.040\right.$, in Fig.4b), $5 \mathrm{~d}\left({ }^{\&} p=0.041\right), 9 \mathrm{~d}\left({ }^{\alpha} p=0.020\right)$ and $11 \mathrm{~d}$ $\left({ }^{\#} p<0.001\right)$, representing that the inflammatory situation of dHAM group was less serious than those of control group at first 11 days after operation.

B. The molecular biological analyses

1) H\&E staining

Normal skin of rats consists of three layers: epidermis, dermis and hypodermis (Fig.5a0). In epidermis (Fig.5a0, A), the most evident cells by H\&E staining were proliferative basal cells, which were considered to be preparing to replace the dead or shed epidermal cells [23]. Situated under the epidermis was the dermis (Fig.5a0, B), which possessed elasticity with various microstructures such as nerves, blood vessels, lymph, sweat glands, hair follicles (Fig.5 a0, blue arrows) and fibers [24]. The hypodermis (Fig.5 a0, C), consisting of adipose tissue and collagen, mainly acted as an energy source [25].

At day 3 (Fig.5 a1 and Fig.6 b11), the wounds in both groups were composed of cells, exudate and inflammatory mediators with huge dissimilarity to normal skins. A great large number of inflammatory cells were produced by the body for antibacteria and preparation to repair the wounds. The dHAM used to cover the wound was still obvious, adhering to the wound compatibly (Fig.6 b12, A). Besides, many granulation tissues (Fig.5 a1, inserted) were found in the deep layer of the wound in control group. When it came to day 7 (Fig.5 a2 and Fig.6 b2), fewer cells under the scabs were found in both groups, implying less inflammation occurred. A porous and loose tissue was 
seen to renew near the normal epidermis. Comparably, fewer cells around the wound in dHAM group reproduced (Fig.6 b2, inserted) than in control group (Fig.5 a2, inserted).

The wound area was becoming smaller with the time passing in both groups of animals. While, the wound surface was covered mostly by epithelial cell layer to form epidermis since 14 days post-operation, and covered completely at day 28 (Fig.5 a3 and Fig.6 b3) which also exhibited from overviewing sight (Fig. 2). In order to observe carefully and clearly, we illustrated detailedly the H\&E staining figures at day 14 and showed as Fig.7. The figure was segmented into three parts, normal tissue neighboring to the original wound (Fig.7, A), the regenerated tissue after the operation (regeneration, Fig.7, B), and the wound which had become scab already (Fig.7 C). The epidermis on the top of the regenerated tissue has been formed while less epidermis was formed on the top of scab parts on animals of both groups. Comparatively, the neogenetic papillary on top of the wound on animals with dHAM treatment was more mature (Fig.7b2, red arrow) than that of the control animals (Fig.7a2, red arrow), on which some scab pieces were dropping off (Fig.7 a3, black arrow). There were fewer cells and blood vessels and more ECM in the regeneration part than those in the scab part. Some neogenetic hair follicles in the regeneration region could be observed (Fig.5a3 and Fig.6b3, blue arrows).

Since the time of 21 days and thereafter (Fig.5 a4-8 and Fig.6 b4-8), the tissue was growing up and the wound was healing gradually. Thus, the generating region became bigger and the scab part became smaller. At day 28, the whole epidermis had 
formed. No fresh dermis was exposed to the air. The biological structure of the generated skin was laminated clearly. The density of cells and ECM became similar to the normal skin. The hypodermis was growing to be more abundant. For the skin of dHAM group animals, more neogenetic hair follicles (Fig.6b4-b8, blue arrows) occupied the regenerated tissue and they were becoming more mature than those of the control animals (Fig.6 a4-a8, blue arrows), though there was still some area with no follicles due to the scar formation.

\section{2) Immunohistochemical detection}

Vascular endothelial growth factor (VEGF) was usually secreted by endothelial cells. Its immunohistochemical staining was commonly used to identify the blood vessels or the potential of neovascularization in the tissues. In the normal rat skin, there exist to be some capillaries at epidermis and dermis layer (Figure.8 a0, black arrows). For the defects of animal skin, more VEGF expression was detected in the wound areas as shown in Fig.8 a1-a2 and b1-b2. Lots of blood vessels were discovered in the deep wound (Fig.8, black arrows in the inserted). Differently, more vascularization occurred at day 7 of dHAM animals and at day 14 of the control animals. From the quantitative analysis, the amount of VEGF expressions of dHAM group was significantly higher at day 7 , but very similar at day 14 to the control animals (Tab.1), which exhibited the consistent results with morphological observation.

Alpha-smooth muscle actin ( $\alpha$-SMA) was usually expressed by smooth muscle cells. Its immunostaining was also often used to identify the smooth muscle (Fig.9 a0, 
blue arrows) and vascular smooth muscle (Fig.9 a0, red arrows) as shown in normal rat skin. From Fig.9 a1-a2 and b1-b2, the expressions of $\alpha$-SMA were clearly detected in great large amount at the whole wound areas, greater at day 7 than at day 14 . However, between the two animal groups, greater $\alpha$-SMA secretion for dHAM species at day 7 than the control species was observed, but at day 14, vice versa. Since day 21 , the difference of $\alpha$-SMA secretion between dHAM and control groups was too little to be negligible. Coincidentally, the neogenetic blood vessels displayed with $\alpha$-SMA immunostaining (Fig.9, red arrows in the inserted) exhibited the similar tendency as smooth muscle staining. The quantitative analyses of IOD values also supported the above results, with values of dHAM group at day 7 was greater but much lower at day 14 than those of control group (Tab.2).

As for transforming growth factor beta-1 (TGF- $\beta 1$ ), it was hardly to detect in normal skin (Fig.10 a0). However, TGF- $\beta 1$ was greatly expressed byepidermal cells (Fig. $10 \mathrm{a} 1$ and b1) and macrophages of neonatal granulation tissue at day 3 (Fig.10a2 and b2). Evidently more granulation tissues were detected in the control group. Thus, the general expression level in control group was higher than that in dHAM group. After 7 days, the amount of TGF- $\beta 1$ reduced quickly. It was hardly to detect after then.

\section{DISCUSSIONS}

Wound healing was contractile and complicated, mainly divided into three phases: inflammation, proliferation and remodeling. The healing began from the 
inflammatory infiltration and epithelial cells growth and migration, and ended by the regeneration of skin appendages and ECM remodeling. Comparing the wound appearances of our two groups of animal skin, the dHAM indicated to promote the contraction of the wound and accelerate the healing process, which might be attributed to the potential of depressing the inflammation at the first several days post-operation. The wound areas of dHAM group were significantly smaller than those of control group at the first 7 days. The results of WBC detection agreed that the inflammation of dHAM group was less serious at $3 \mathrm{~d}(p=0.040), 5 \mathrm{~d}(p=0.041), 9 \mathrm{~d}$ ( $p=0.020)$ and $11 \mathrm{~d}(p<0.001)$, which verified that dHAM truly alleviated the inflammation of the wound. It might relate to the decreasing secretion of TGF- $\beta 1$ in macrophages of neonatal granulation tissues, as our detection in the following experiments.

H\&E staining helped to show the microscopic observation of the healing progress of the wounds at various time points. Since the 3rd day, the healing started from a great number of cells with the secretion of exudate followed by the formation of scabs. In dHAM group, dHAM clung to the wounds just like a bed for the newborn cells to adhere and to migrate around, which was beneficial for the tissues to form a protective skin epidermis on the top of wound. Neonatal epidermis appeared at day 7 and extended to the wound center in the following days. These results explained why the healing took place accompanied with the wound contractile, as we observed from our sight-seeing (Fig. 2). Until day 14, the cells became fewer in number and sparser in space like the normal tissue, which was comparatively more apparent in dHAM 
group than in control group (Fig.7). And more mature epidermis formed in dHAM group than in control group. Meanwhile, the appendages of skin like blood vessels and hair follicles regenerated. A large amount of bloods vessels exhibited mainly in granulation tissues at initial phase and then gradually disappeared at remodeling phase. The hair follicles began to be observed at around 14 days in the regenerated skin (Fig.5 a3 and Fig.6 b3, blue arrow), with the larger regeneration part and the smaller scab area after then. However, the follicle formation seemed quite tardy. Until 8 mon, there was still a small X-shape scar with no hair follicles, which was considered to be in the center of the original wound (Fig.5 a8 and Fig.6 b8). The healing situation was being followed and detected at longer repairing time in our group.

VEGF, acting as a symbol factor of blood vessels, could promote angiogenesis and enhance endothelial cell proliferation and migration [26, 27]. It also had influences on wound closure and repairing, including the formation of granulation tissues. But every coin has two sides. High-level expressions of VEGF could lead to overabundant scar formation [28]. Our results showed that the amount of VEGF in dHAM group was higher than that in control group at 7 days (Fig.8, Tab.1). This demonstrated that dHAM improved the level of VEGF, which was helpful to form more blood vessels to provide nutrition so that it could accelerate the process of wound repairing. This was positive for wound healing within a short time though the increased expression might cause the final scar formation. Expectedly, VEGF secretion of dHAM animal reduced to the similar value to that of the control animal at day 14. 
During wound healing and skin regeneration, $\alpha$-SMA secreted by myofibroblasts helped to contract the distance among cells to contribute to the closure of the wound [29,30]. In addition, myofibroblasts played an important role in wound healing by secreting a variety of cytokines and growth factors as well as ECM and its degradative enzymes, which took part in remodeling phase of healing steps [30,31]. In our study, high expressions of $\alpha$-SMA were shown in both groups at 7 days, significantly more in dHAM group (Fig.9, Tab.2). This demonstrated that dHAM improved the level of a-SMA, which was beneficial to wound contraction and vessel angiogenesis. At day 14 , the $\alpha$-SMA amount of dHAM animal decreased, which was significantly lower than that in control animal. This indicated that the expressions of $\alpha$-SMA in dHAM group reduced more quickly than the control group, leading to reduce angiogenesis of granulation tissues and thus to cause fewer scabs.

TGF- $\beta 1$ was the key growth factor in the early stage of wound healing. It was capable to promote VEGF expressions at the site of wound. Besides, it was conducive to trans-differentiate the fibroblastic cells into the myofibroblasts that secreted $\alpha$-SMA to contract the wound as mentioned earlier [30-32]. But excessive TGF- $\beta 1$ was a vital cause of scar formation due to the accumulation of collagens [33]. At day 3, the amount of TGF- $\beta 1$ expression was higher in control group, which accounted for the more serious inflammatory in control group than in dHAM group (Fig. 10). It could be inferred that dHAM decreased the secretion of TGF- $\beta 1$ relatively, thereby alleviating the inflammation and relieved the scar formation. 


\section{CONCLUSIONS}

In this work, dHAM was applied to repair the full-thickness skin defects using SD rats as the experimental animals. dHAM was a biocompatible wound dressing with good qualities in wound repairing. It accelerated and strengthened the process of the wound self-healing. In our experiment, dHAM increased the expression level of VEGF, which played a role mainly in proliferation phase to promote the skin regeneration by neovascularization. And, dHAM helped to increase the level of a-SMA secretion, which involved in remodeling phase like contraction and ECM remodeling. Besides, it also alleviated the inflammation of wounds at the first several days post-operation, and thus relieved the scar formation via decreasing the excessive level of TGF- $\beta 1$, which acted in primary inflammation phase as well as induced the biosynthesis of VEGF and $\alpha$-SMA. Together with the advantages of anti-inflammatory, no antigen, feasibility, tolerance and low cost, we believed that the dHAM was a promising wound dress to promote skin repairing at early stage and further advance the skin regeneration in general. More researches remained to go far to discover the thorough mechanism and to try the application in clinic in future.

\section{ACKNOWLEDGEMENTS}

The authors gratefully acknowledge the financial support from the National Science Foundation (81471797) and Project of Scientific Innovation Team of Ningbo (2015B11050, 2014B82002 and 2012B82019), China. This work was also sponsored by K.C. Wang Magna/Education Fund of Ningbo University. 


\section{CONFLICT OF INTEREST}

The authors declare no competing financial interest.

\section{REFERENCES}

[1] H.Bi, Y.Jin, Current progress of skin tissue engineering: Seed cells, bioscaffolds, and construction strategies, Burns Trauma1 (2013) 63-72.

[2] J.Harder, J. M.Schröder, R.Gläser, The skin surface as antimicrobial barrier: present concepts and future outlooks, Exp Dermatol 22 (2013) 1-5.

[3] D. H.Hu, Y. C.Wang, Lay more stress on the study and application of covering materials for wounds, Zhonghua Shao Shang Za Zhi 28 (2012) 323-326.

[4] E. S.Sheikh, E. S.Sheikh, D. E.Fetterolf, Use of dehydrated human amniotic membrane allografts to promote healing in patients with refractory non healing wounds, Int Wound J 11 (2014) 711-717,.

[5] S.Dhivya, V. V.Padma, E.Santhini, Wound dressings - a review, Biomedicine 5 (2015) 22.

[6] C.Mihail, M.Erika, E. A.Farkashet al., Bioengineered self-assembled skin as an alternative to skin grafts,Plast Reconstr Surg Glob Open4(2016) e731.

[7] S. C.Yu,Y. Y.Xu, Y.Liet al., Construction of tissue engineered skin with human amniotic mesenchymal stem cells and human amniotic epithelial cells,Eur Rev Med Pharmacol Sci 19 (2015) 4627-4635.

[8] M.Parthasarathy,R.Sasikala, P.Gunasekaranet al., Antimicrobial activity of human amniotic and chorionic membranes,An. Fac. Med., Univ. Nac. Mayor San 
Marcos Lima 2 (2014) 19-24.

[9] H.Tauzin,G.Rolin, C.Viennetet al., A skin substitute based on human amniotic membrane,Cell Tissue Bank 15 (2014) 257-265,.

[10]M.Gholipourmalekabadi, M.Sameni, D.Radenkovic et al., Decellularized human amniotic membrane: how viable is it as a delivery system for human adipose tissue-derived stromal cells. Cell Prolif 49 (2016) 115-121.

[11]P. Shi, M. Gao, Q. Shen, L. Hou, Y. Zhu, J. Wang, Biocompatible surgical meshes based on decellularized human amniotic membrane, Mater Sci Eng C Mater Biol Appl 54 (2015) 112-119.

[12]S. M.Zidan,S. A.Eleowa, M. A.Nasefet al., Maximizing the safety of glycerol preserved human amniotic membrane as a biological dressing, Burns 41 (2015) 1498-1503.

[13] Y.Jie, W.Li, J.Huang et al., Transplantation of human adipose stem cell-derived hepatocyte-like cells with restricted localization to liver using acellular amniotic membrane,Stem Cell Res Ther 6 (2015) 1-14.

[14] J.Halper, M.Kjaer, Basic components of connective tissues and extracellular matrix: elastin, fibrillin, fibulins, fibrinogen, fibronectin, laminin, tenascins and thrombospondins, Adv Exp Med Biol802 (2014) 31-47.

[15]I.Senyürek,W. E.Kempf, G.Klein et al., Processing of laminin $\alpha$ chains generates peptides involved in wound healing and host defense, J Innate Immun6 (2014) 467-484.

[16]T. J.Koob,J. J.Lim, N.Zabeket al., Cytokines in single layer amnion allografts 
compared to multilayer amnion/chorion allografts for wound healing, $J$ Biomed Mater Res B Appl Biomater103 (2015) 1133-1140.

[17]N. H.Riordan, B. A.George, T. B.Chandler et al., Case report of non-healing surgical wound treated with dehydrated human amniotic membrane, $J$ Transl $\operatorname{Med13}(2015)$ 1-5.

[18]T.Gambichler, A.Pljakic, L.Schmitz, Recent advances in clinical application of optical coherence tomography of human skin,Clin Cosmet Investig Dermatol 8 (2015) 345-354.

[19]R. F.Pereira,C. C.Barrias, P. L.Granjaet al., Advanced biofabrication strategies for skin regeneration and repair, Nanomedicine 8 (2013) 603-621.

[20]E.Mahdi,A.Mina, R.Soghraet al., The healing effect of amniotic membrane in burn patients, World J Plast Surg5 (2016) 39-44.

[21]D.J. Loeffelbein, C. Baumann, M. Stoeckelhuber, R. Hasler, T. Mucke, L. Steinstraßer, E. Drecoll, K-D Wolff, M.R. Kesting. Amniotic membrane as part of a skin substitute for full-thickness wounds: An experimental evaluation in a porcine model. J Biomed Mater Res 100B (2012) 1245-1256.

[22]C.Gong, L.Hou, Y.Zhu et al., In vitro constitution of esophageal muscle tissue with endocyclic and exolongitudinal patterns, ACS Appl Mater Interfaces 5 (2013) 6549-6555.

[23]A. M.Tadeu, V.Horsley, Epithelial stem cells in adult skin,Curr Top Dev Biol 107C (2014) 109-131.

[24]F. R.Fernandes,N. R.Smyth, O. L.Muskenset al., Interactions of skin with gold 
nanoparticles of different surface charge, shape, and functionality, Small 11 (2015) 713-721.

[25]B. K.Sun,Z.Siprashvili, P. A.Khavari, Advances in skin grafting and treatment of cutaneous wounds, Science346 (2014) 941-945.

[26]Q. L.Tang,S. S.Han, J.Fenget al., Moist exposed burn ointment promotes cutaneous excisional wound healing in rats involving VEGF and bFGF,Mol Med Rep 9 (2014) 1277-1282.

[27]X.Liu, R.Shen, M.Bian et al., Expressions of VEGF, PDGF and bFGF inwoundcutaneoustissue and theirsignificanceinrats, Acta Acad. Med. Qingdao Univ.52 (2016) 209-211.

[28]K. E.Johnson, T. A.Wilgus, Vascular endothelial growth factor and angiogenesis in the regulation of cutaneous wound repair, Adv Wound Care 3(2014) 647-661.

[29] N.Yoshiba,K.Yoshiba, N.Ohkura et al., Immunohistochemical analysis of two stem cell markers of $\alpha$-smooth muscle actin and STRO-1 during wound healing of human dental pulp,Histochem Cell Biol 138 (2012) 583-592.

[30]N.Yoshiba,K.Yoshiba, N.Ohkura et al., Correlation between fibrillin-1 degradation and mRNA downregulation and myofibroblasts differentiation in cultured human dental pulp tissue, J Histochem Cytochem 63 (2015) 438-448.

[31]L.Micallef, N.Vedrenne, F.Billet et al., The myofibroblast, multiple origins for major roles in normal and pathological tissue repair, Fibrogenesis Tissue Repair 5 (2012) 1-5.

[32]R. W. D.Gilbert,M. K.Vickaryous, A. M.Viloria-Petit, Signalling by transforming 
growth factor beta isoforms in wound healing and tissue regeneration, $J$ Dev Biol 4 (2016) 21.

[33] S. W.Lu,X. M.Zhang, H. M.Luo et al.,Clodronate liposomes reduce excessive scar formation in a mouse model of burn injury by reducing collagen deposition and TGF- $\beta 1$ expression, Mol Biol Rep 41 (2014) 2143-2149. 


\section{Captions:}

Fig.1 Full-thickness skin defects on the back of SD rats. (a) Wound appearance immediately after surgery. (b) dHAM adhered onto the left wound (arrow); the right wound was set as the control.

Fig.2 Overview of wound on the rat back at $7 \mathrm{~d}$ (a1), $14 \mathrm{~d}$ (a2), $21 \mathrm{~d}(\mathrm{a} 3)$ and $28 \mathrm{~d}$ (a4). Arrow points to animals with dHAM treatment.

Fig.3 Wound area (\%) as the function of time (days). Curves displaying the area alteration tendency (a), and differences with statistical analyses between dHAM group and control group (b). ${ }^{*} p=0.018,{ }^{\&} p=0.024,{ }^{\alpha} p=0.010 . p<0.05$ was considered to be significant.

Fig.4 Curves of white blood cells (WBC, \%) in full blood with the time (days) post-operation (a), and differences with statistical analyses between dHAM group and control group (b). ${ }^{*} p=0.040,{ }^{\&} p=0.041,{ }^{\alpha} p=0.020,{ }^{\#} p<0.001 . p<0.05$ was considered to be significant.

Fig.5 H\&E staining of normal skin (a0), and skins of control group at $3 \mathrm{~d}(\mathrm{a} 1), 7 \mathrm{~d}$ (a2), 14 d (a3), 21 d (a4), 28 d (a5), 42 d (a6), 56 d (a7) and 8 mon (a8). Arrow points to neogenetic hair follicles. A- epidermis layer, B- dermis layer, C- hypodermis layer. Scale bar, $200 \mu \mathrm{m}$. Inserted pictures, scale bar, $50 \mu \mathrm{m}$.

Fig.6 H\&E staining of skins of dHAM group at 3 d (b11, b12), 7 d (b2), 14 d (b3), 21 d (b4), 28 d (b5), 42 d (b6), 56 d (b7) and 8 mon (b8). Arrow points to neogenetic hair follicles. A- dHAM. Scale bar, $200 \mu \mathrm{m}$ (except b12, scale bar, $50 \mu \mathrm{m}$ ). Inserted pictures, scale bar, $50 \mu \mathrm{m}$. 
Fig.7 H\&E staining of skins at $14 \mathrm{~d}$. a- control group, b- dHAM group. The whole skin, the wound and periphery, was divided into 3 parts; A- normal part, Bregenerated part, C- scab part. I- epidermis layer, II- dermis layer.a1, b1-scale bar, $200 \mu \mathrm{m} ; \mathrm{a} 2, \mathrm{a} 3, \mathrm{~b} 2 \&$ b3- scale bar, $50 \mu \mathrm{m}$. Red arrows in a2 \& b2 point to papillary layer and in a3 to the dropping scab pieces.

Fig.8 VEGF immunostaining. VEGF is expressed by endothelial cells and VEGF immunostaining is used to identify blood vessels (brown color). a0- normal skin. acontrol group and b- dHAM group; $1-7$ days and 2- 14 days. The inserted is the magnification of circle region of the figures. Scale bar, $200 \mu \mathrm{m}$. Scale bar of inserted pictures is $50 \mu \mathrm{m}$.

Fig.9 $\alpha$-SMA immunostaining. $\alpha$-SMA is secreted by smooth muscle cells. $\alpha$-SMA immunostaining is used to identify vascular smooth muscle (red arrows, brown color) and smooth muscle (blue arrows, brown color) .a0- normal skin. a- control group and b- dHAM group; 1- 7 days and 2- 14 days. The inserted is the magnification of circle region of the figures. Scale bar, $200 \mu \mathrm{m}$. Scale bar of inserted pictures is $50 \mu \mathrm{m}$.

Fig.10 TGF- $\beta 1$ immunostaining. Its immunostaining (brown color) is indicated by arrows a0- normal skin (scale bar $200 \mu \mathrm{m}$ ). a1, a2- control group at day 3 (scale bar, $50 \mu \mathrm{m}$ ); b1, b2- dHAM group at day 3 (scale bar, $50 \mu \mathrm{m}$ ). 
Tab.1 Integrated option density (IOD) of VEGF. Difference at day 7 shows significance $(p=0.003)$ * Values indicated as mean \pm standard deviation (SD). * Statistical analyses were performed with IBM SPSS Statistics 19. $p<0.05$ was considered to be significant.

\begin{tabular}{|c|c|c|c|}
\hline Group & n & day 7* & day 14 \\
\hline dHAM & 3 & $26192.80 \pm 4754.39$ & $16913.42 \pm 7257.46$ \\
\hline Ctrl & 3 & $7026.08 \pm 1414.40$ & $11881.68 \pm 6706.61$ \\
\hline \multicolumn{2}{|c|}{ t-value $^{\#}$} & 6.693 & 0.882 \\
\hline \multicolumn{2}{|c|}{-value $^{\#}$} & 0.003 & 0.428 \\
\hline
\end{tabular}

Tab.2 Integrated option density (IOD) of $\alpha$-SMA. Differences at day 7 and day 14 show significance $\left({ }^{\alpha} p=0.016,{ }^{\&} p=0.043\right) .{ }^{*}$ Values indicated as mean \pm standard deviation (SD). ${ }^{\text {\# }}$ Statistical analyses were performed with IBM SPSS Statistics 19. $p<0.05$ was considered to be significant.

\begin{tabular}{|c|c|c|c|}
\hline Group & $\mathbf{n}$ & day $\mathbf{7}^{\alpha, *}$ & day 14 \\
\hline dHAM & 3 & $94410.75 \pm 12163.94$ & $1319.54 \pm 621.53$ \\
\hline Ctrl & 3 & $48825.58 \pm 15384.82$ & $17550.92 \pm 6137.98$ \\
\hline \multicolumn{2}{|c|}{ t-value $^{\#}$} & 4.026 & -4.557 \\
\hline \multicolumn{2}{|c|}{ p-value $^{\#}$} & 0.016 & 0.043 \\
\hline
\end{tabular}

